\title{
A multi-wavelength study of solar coronal-hole regions showing radio enhancements
}

\author{
T. Moran ${ }^{1}$, N. Gopalswamy ${ }^{1}$, I. E. Dammasch ${ }^{2}$, and K. Wilhelm ${ }^{2}$ \\ 1 Center for Solar Physics and Space Weather, The Catholic University of America, Washington, DC 20064 \\ and NASA Goddard Space Flight Center, Code 682.3, Greenbelt, MD 20771, USA \\ 2 Max-Planck-Institut für Aeronomie, Max-Planck-Str. 2, 37191 Katlenburg-Lindau, Germany
}

Received 8 January 2001 / Accepted 9 April 2001

\begin{abstract}
We observed $17 \mathrm{GHz}$ microwave-enhanced regions in equatorial coronal holes (ECH) together with extreme-ultraviolet (EUV), far-ultraviolet (FUV) and visible emissions in a search for temperature increases which might explain the bright spots in radio wavelengths. The ultraviolet (UV) observations span a wide range of formation temperatures $(8000 \mathrm{~K}$ to $630000 \mathrm{~K})$. Increased UV emission was observed at the approximate location of the radio enhancements, but unlike the radio brightening, the UV emission did not exceed the mean quiet sun level. However, there were two observations showing increased $\mathrm{H} \alpha$ brightness in radio enhancements above mean quiet sun levels. No $\mathrm{H} \alpha$ bright spots were detected in ECHs outside of radio enhancement regions. The ECH $\mathrm{H} \alpha$ bright spots were caused by bright fibrils, bright points and a lack of dark fibrils. Since the $17 \mathrm{GHz}$ and $\mathrm{H} \alpha$ enhancements are co-spatial, have equal integrated normalized enhanced emission and brightness temperatures, the observations suggest that the radio enhancements are caused by increased fibril radio emission. In addition, increased Fe XII EUV emission was recorded at the location of some well-defined radio enhancements, which were the bases of coronal plumes. Since the radio brightness temperature is much lower than the Fe XII formation temperature, the radio and EUV enhancements are likely both related to the presence of concentrated magnetic flux, but do not arise from the same physical layer.
\end{abstract}

Key words. Sun: radio radiation - Sun: UV radiation - Sun: chromosphere - Sun: transition region - Sun: corona

\section{Introduction}

The solar structures known as coronal holes $(\mathrm{CH})$ are important for understanding the Sun's effects on the Earth, since they are the source of the high-speed solar wind (Krieger et al. 1973). These regions have lower coronal densities and temperatures than non-hole regions, which is presumably due to open magnetic field lines in the hole. $\mathrm{CHs}$ can be identified by a decrease in coronal line emission, for example the EUV Fe XII $195 \AA$ line observed by the Solar and Heliospheric Observatory Extreme-ultraviolet Imaging Telescope (SOHO/EIT) instrument (Delaboudinière et al. 1995), or in emission from lines which are excited from the corona, such as the near infrared He $10830 \AA$ line. One unexplained aspect of $\mathrm{CHs}$, both near the poles and the equator, is the presence of bright regions in radio emission in some holes (Kundu \& McCullough 1972; Wefer \& Bleiweiss 1976; Evanov et al. 1980; Kosugi et al. 1986; Shibasaki 1998; Gopalswamy et al. 1999a,b; Nindos et al. 1999; Pohjolainen 2000).

Send offprint requests to: T. Moran, e-mail: tmoran@cspsw4.nascom.nasa.gov
These radio enhancements may be related to the physical conditions in the hole which give rise to the fast solar wind.

These regions were first detected in polar coronal holes $(\mathrm{PCH})$ and later in equatorial coronal holes $(\mathrm{ECH})$, which often begin as PCHs and move to low latitudes. Brightenings in $\mathrm{CHs}$ have been observed at frequencies between 17 and $87 \mathrm{GHz}$. These enhancements may be classified as compact, $1^{\prime}$ or less in extent, or diffuse, extending over the entire $\mathrm{CH}$. Observations at 17 and $36 \mathrm{GHz}$ made with $10-45^{\prime \prime}$ spatial resolution show both diffuse and compact enhancement within polar and equatorial $\mathrm{CHs}$, with the mean emission level exceeding that of the quiet sun (Kosugi et al. 1986; Shibasaki 1998; Gopalswamy et al. 1999a,b; Nindos et al. 1999). Observations at $87 \mathrm{GHz}$ show the presence of enhanced compact regions surrounded by depressed diffuse emission in CH's at a resolution of $1^{\prime \prime}$ (Pohjolainen et al. 2000; Pohjolainen 2000). At $10.7 \mathrm{GHz}$, observations made with a resolution of $1.25^{\prime \prime}$ show the mean polar $\mathrm{CH}$ levels are depressed relative to the quiet sun (Furst \& Hirth 1975), but small unresolved enhanced emission sources might be present. Observations 
at $98 \mathrm{GHz}$ made with a resolution of $17^{\prime \prime}$ show no polar CH diffuse or compact enhancements (Kosugi et al. 1986). To summarize, the mean $\mathrm{CH}$ emission level is depressed between 10.7 and $98 \mathrm{GHz}$, and compact enhanced regions have been observed between 17 and $87 \mathrm{GHz}$. High resolution observations outside this frequency range are required in order to determine the frequency extent of compact enhancements.

We consider $\mathrm{CH}$ radio emission at $17 \mathrm{GHz}$, since compact enhancements are strong at this frequency and the Nobeyama Radioheliograph observes the sun constantly at $17 \mathrm{GHz}$ with $1 \mathrm{~s}$ time resolution (Nakajima et al. 1995). Full disk $17 \mathrm{GHz}$ solar images at ten minute intervals are readily available from the Nobeyama Radio Observatory web server. At $17 \mathrm{GHz}$, the diffuse component is characterized by temperatures typically $1000 \mathrm{~K}$ above the quiet Sun (QS) level of $10000 \mathrm{~K}$, while the compact component is as much as $3000 \mathrm{~K}$ above the background. The polar brightness enhancements are generally greater than the equatorial enhancements, due to contributions from microwave limb brightening. The true brightness temperature increase is in fact larger if the compact bright regions are unresolved.

Comparison of microwave images and longitudinal magnetograms shows that enhancements are correlated with regions of strong unipolar magnetic flux (Gopalswamy et al. 1999a,b, 2000) and with enhanced coronal emission in plume bases (Pohjolainen 2000). Enhancements are not found in areas of bipolar or mixed flux. Radio image sequences with ten minute cadence show the enhancements are highly variable on scales of ten minutes or less (Gopalswamy 1999b). Increased limb brightening has also been found in PCHs for some FUV emission lines (C III, O IV, and S V) (Wilhelm et al. 1998). The source of the enhanced radio emission has been investigated through modeling of the chromospheric, transition region and coronal contributions. We define the chromospheric temperature to be between $4000 \mathrm{~K}$ and $20000 \mathrm{~K}$, the transition region between $20000 \mathrm{~K}$ and $500000 \mathrm{~K}$ and the quiet coronal temperature between $500000 \mathrm{~K}$ and $2000000 \mathrm{~K}$, and the lower, middle and upper chromospheric temperature ranges to be $4000 \mathrm{~K}$ to $8000 \mathrm{~K}$, $8000 \mathrm{~K}$ to $12000 \mathrm{~K}$ and $12000 \mathrm{~K}$ to $20000 \mathrm{~K}$, respectively. Since the optical depth is proportional to the line-of-sight integral of $n_{\mathrm{e}}^{2} / T_{\mathrm{e}}^{3 / 2}$, where $n_{\mathrm{e}}$ is the electron density and $T_{\mathrm{e}}$ is the electron temperature, the $\mathrm{CH}$ contribution to the total $17 \mathrm{GHz}$ emission must be small, approximately 1 to $2 \%$, due to the low densities and high temperatures in the corona (Gopalswamy et al. 1998, 1999a,b). The contribution from the transition region between $20000 \mathrm{~K}$ and $200000 \mathrm{~K}$ must be negligible as well, since radio emission models excluding that region agree with the observed QS spectrum (Grebinskii 1987). Thus, the radio enhancements must be formed at temperatures below $20000 \mathrm{~K}$, since temperatures above $200000 \mathrm{~K}$ are precluded due to low optical depth. This is consistent with the measured $17 \mathrm{GHz}$ brightness temperature of $10000 \mathrm{~K}$ for the QS and peak temperatures of $12000 \mathrm{~K}$ to $13000 \mathrm{~K}$ for the compact enhancements.

In order to determine the cause of the enhancements, we have made a series of observations in a search for the physical layer where the enhanced radiation is formed. If the cause of the radio enhancement in polar and equatorial holes is a difference in temperature profile between the QS and $\mathrm{CH}$ regions of strong magnetic flux, the intensities of UV lines emitted at the radio formation height will also be affected, since the UV line excitation functions are temperature dependent. A correlation between radio and UV line intensity in an ECH radio enhancement would indicate the height and temperature of the increased $17 \mathrm{GHz}$ emission. Previous observations have shown that some upper chromospheric emission lines, such as $\mathrm{H}$ I Ly $\alpha$, Ly $\beta$, Ca II K and Mg II K, which form at temperatures below $20000 \mathrm{~K}$, are slightly more intense in $\mathrm{CHs}$ (Bocchialini \& Vial 1996), but the characteristics of these lines in ECH radio enhancement regions have not been measured. Therefore, a series of UV ECH observations were made for the first time using the Solar Ultraviolet Measurements of Emitted Radiation (SUMER) telescope and spectrograph (Wilhelm et al. 1997) on board the Solar and Heliospheric Observatory (SOHO) (Domingo et al. 1995) in several FUV chromospheric and transition region lines. The spectral line observations covered a formation temperature range of 8000 to $630000 \mathrm{~K}$, which includes the lower chromosphere through the upper transition region to the lower corona. In this article, we present the results of a comparison between UV and microwave observations in these CHs. In addition, we compare radio images with visible $\mathrm{H} \alpha$ images to include the lower and middle chromosphere in the enhancement layer search.

\section{Observations}

Two ECHs were observed with SOHO/SUMER on seven days during October and November of 1999. A total of 12 FUV lines were recorded during the passage of the holes across the disk in October and November of 1999 as part of SOHO Joint Observing Program (JOP) 115. Full-disk radio, $\mathrm{H} \alpha$ and EUV observations were made synoptically during all of the SUMER observations. The difractionlimited resolution for the radio images is $10^{\prime \prime}$ for observations made within 2 hours of local noon. Outside this interval resolution decreases due to a decrease in projected array length. The effective resolution will also depend upon the image construction, which introduces artifacts (Nindos et al. 1999). The images collected from the Nobeyama web server appear to have a resolution of $\sim 18^{\prime \prime}$, since there are no features in the images with smaller length scales. Images at visible and UV wavelengths include features with dimensions at their resolution limits of $1-4^{\prime \prime}$, so it seems unlikely that $17 \mathrm{GHz}$ features would be limited to length scales above $18^{\prime \prime}$, unless their apparent size is limited by resolution. Radio images are provided in a 512 by 512 frame at $4.91^{\prime \prime} /$ pixel. $\mathrm{H} \alpha$ images at line center are recorded daily at the Big Bear Solar 
Observatory (BBSO) and provided in an 1889 by 1889 frame at $1.07^{\prime \prime}$ per pixel and $1^{\prime \prime}$ to $2^{\prime \prime}$ resolution. Full-disk images in Fe XII $195 \AA$ emission are recorded daily by EIT on board the SOHO spacecraft with a resolution of $2^{\prime \prime}$ and provided in a 1024 by 1024 frame at $2.6^{\prime \prime} /$ pixel. The Fe XII images are used to identify $\mathrm{CHs}$ and map their boundaries. Full-disk longitudinal magnetograms were recorded daily by the Michelson Doppler Imager (MDI) on board SOHO and provided in a 512 by 512 frame at 3.9" / pixel.

The FUV observations with SUMER in several chromospheric and transition region lines included portions of two ECHs and the surrounding areas (Wilhelm et al. 2001). The slit used was one arcsecond wide and either $120^{\prime \prime}$ or $300^{\prime \prime}$ long, and was scanned across the solar surface. A sub-frame image was constructed from the individual slit position measurements. Individual exposure times were $150 \mathrm{~s}$ and total exposure times were 4 to 6 hours. The regions observed varied from 100 to $300^{\prime \prime}$ in width, with a height of either 120 or $300^{\prime \prime}$. All of the observations considered except one included area both inside and outside the $\mathrm{ECH}$ and all of the observations included regions of radio enhancement. The dates of the observations, ion species, wavelengths of the lines and their formation temperatures $\left(T_{\mathrm{f}}\right)$ are listed in Table 1 . The ionization states range from neutral $\left(\mathrm{H}^{0}, \mathrm{O}^{0}\right)$ to $\mathrm{Ne}^{7+}$, and formation temperatures from $10000 \mathrm{~K}$ to $630000 \mathrm{~K}$. Since lines are formed over a range of temperature, $T_{\mathrm{f}}$ indicates the mean. The formation temperature limits for a given line are determined by the ionization potentials, and excitation energies. The table also notes the type of region contained in the field of view such as QS and $\mathrm{CH}$, the difference in ground and excited state energies, $\chi$, and a factor $\alpha$, which will be discussed in Sect. 3 .

Radio enhancements were present in five of the ECH observations. The outline of each $\mathrm{CH}$ was determined from the EIT FexII $195 \AA$ image. There were small, welldefined ECH radio enhancements of 15 to $20 \%$ peak in the SUMER field of view ranging in size from $20^{\prime \prime}$ to $50^{\prime \prime}(F W H M)$ on October 24 and 29, 1999, as well as larger diffuse radio enhancements of 5 to $10 \%$ peak on three other observation days. The well-defined enhancements are found in regions of strong unipolar magnetic flux, consistent with previous observations (Gopalswamy et al. 1999a,b). Since the bright features are probably not resolved at the $18^{\prime \prime}$ to $20^{\prime \prime}$ resolution of the radioheliograph, the true enhancement is probably larger and therefore the temperature increase in the enhancement is at least 15 to $20 \%$ above the QS level.

Images of an ECH in $17 \mathrm{GHz}$ radio, FexII and $\mathrm{H} \alpha$ brightness and the magnetogram recorded on October 24, 1999 are shown in Figs. 1a-d. SUMER UV observations were made from 00:00 to 06:09 UT. The radio image was obtained on October 24, 03:06 UT, within the SUMER observing interval. The Fe XII and $\mathrm{H} \alpha$ images and the MDI magnetogram were recorded on October 23 at 22:24, 15:26 and 13:02 UT, respectively. The Fe XII and $\mathrm{H} \alpha$ data and the magnetogram were binned to match the radio image scale of $4.91^{\prime \prime} /$ pixel. The $\mathrm{H} \alpha$ image has also been smoothed

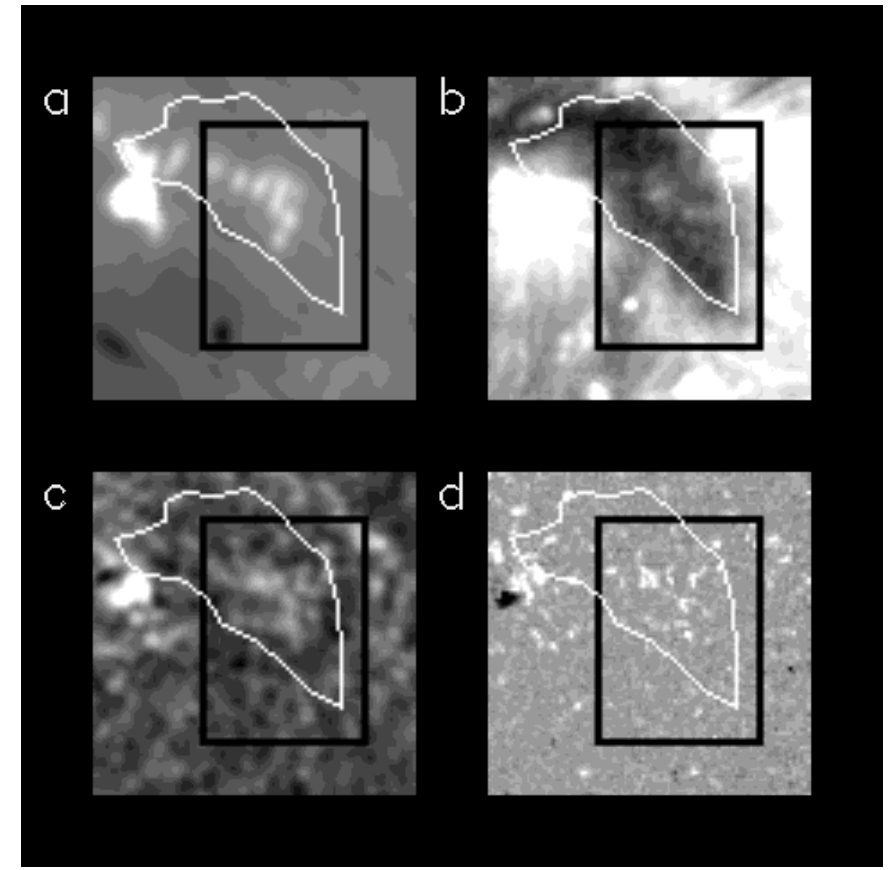

Fig. 1. Images of the October 24, $1999 \mathrm{ECH}$ in a) $17 \mathrm{GHz}$ radio, b) $195 \AA$ (EIT Fe XII), c) H $\alpha$ emission, and d) the corresponding $\mathrm{SOHO} / \mathrm{MDI}$ magnetogram. The $\mathrm{CH}$ boundary is outlined in white and the SUMER field of view is outlined in black.

with a $4 \times 4$ box function, which results in an effective resolution of $19.6^{\prime \prime}$, comparable to the effective radio resolution of $18^{\prime \prime}$

The boundaries of the ECH and SUMER field of view $\left(391^{\prime \prime}\right.$ to $553^{\prime \prime} \mathrm{W}, 0^{\prime \prime}$ to $\left.300^{\prime \prime} \mathrm{N}\right)$ are outlined in each image. Note the chain of bright regions within the hole boundary and SUMER field of view in the radio image. The particular Fe XII and BBSO $\mathrm{H} \alpha$ images and MDI magnetograph considered for comparison were obtained closest in time to the UV observations. Ideally, comparisons should be made with simultaneous observations, but none were available. However, bright features present in the radio image at 03:06 UT on Oct. 24, during the UV observations, were clearly visible in the $\mathrm{H} \alpha$ image obtained at 15:26 UT on Oct. 23, 12 hours earlier, and in the magnetogram obtained at 13:02 UT on Oct. 23, 14 hours earlier. In addition, the bright features were present in the radio images until the end of the observing day on Oct. 24, at 05:30 UT. Thus the features had a lifetime of at least 16.5 hours. These areas coincide generally with regions of strong unipolar flux and enhanced $\mathrm{H} \alpha$ and Fe XII emissions. The magnetic fields measured in these regions with MDI had the largest strengths seen in the ECH enhancements, $150 \mathrm{G}$. These results are consistent with the previous analysis of radio and $\mathrm{H} \alpha$ emission in $\mathrm{CHs}$ by Gopalswamy (2000), who first pointed out the association of microwave radio and $\mathrm{H} \alpha$ enhancements in ECHs.

The October 23 and 29 observations both show that some of the radio enhancements are the bases of equatorial coronal plumes as recorded by EIT at $195 \AA$. This is 
consistent with observations of polar plumes, which showed that those plumes originate from strong unipolar flux concentration (DeForest et al. 1997). In addition to having the same magnetic field correlation, radio enhancements and plumes also have similar time variability, with both showing strong intensity fluctuations on time scales of ten minutes or less (for radio variability, see Gopalswamy et al. 1999, for plume variability, see DeForest et al. 1997). Thus, there seems to be a strong link between radio enhancements and equatorial coronal plumes. One significant difference between polar and equatorial plumes is the location of the radiant, or point of apparent origin of the plumes within the sun. Polar plumes appear to originate from a radiant which is approximately $0.6 R_{\mathrm{O}}$ from the disk center, where $R_{\mathrm{O}}$ is the solar radius (Fisher \& Guhathakurta 1995), while the equatorial plumes observed on October 23, 1999 appear to radiate from a point much closer to the surface, approximately $0.9 R_{\mathrm{o}}$. This effect may be partially caused by strong flux concentrations outside the ECH, such as the bipolar structure bordering the northeast boundary of the hole.

Sub-frames of the October 24 radio emission and $\mathrm{H} \alpha$ brightness in the SUMER field of view are shown in Figs. 2a,b, the corresponding magnetogram is shown in Fig. 2c and the SUMER O i $948.7 \AA$, H i Ly $4949.7 \AA$ and He II $958.6 \AA$ frames are shown in Figs. 2d-f. Figure 3 shows a plot of normalized radio, $\mathrm{H} \alpha, \mathrm{H}$ i Ly 4 and He II brightness along Col. 20, which is marked with a vertical dash at the bottom of each frame. The boundary of the ECH is marked with a bar on the abscissa. The O I emission followed the H I Ly 4 emission to within $4 \%$. The enhanced radio emission peaks at 1.2 , while the reduced resolution $\mathrm{H} \alpha$ emission peaks at 1.3. Since the radio resolution element may be larger than the 18 arcsec assumed, the $\mathrm{H} \alpha$ contrast might equal the radio contrast if both observations were made with the same resolution.

The UV line emission is slightly lower in the ECH than in the quiet sun, but increases near the radio and $\mathrm{H} \alpha$ peaks to quiet sun levels. Thus, the upper chromosphere and transition region in the radio enhancements appears to be hot relative to the ECH outside the enhancements, but not relative to the quiet sun. This suggests that the mechanism responsible for radio enhancements also affects the chromosphere and transition region. The difference in location between the radio, $\mathrm{H} \alpha$ and UV emission peaks may be due in part to the spreading of field lines with height. In the case of the UV emission, observations have shown that the vertical scale height is increased inside polar coronal holes (Huber et al. 1974), which is likely true for ECHs as well. In addition, the radio, $\mathrm{H} \alpha$ and UV observations were not made simultaneously, so the network structure could have changed slightly between measurements.

To summarize the primary results, we compared radio emission in ECH regions showing enhancements with FUV, H $\alpha$ and EUV Fe XII brightness. There was no increased SUMER UV line emission, as compared to quiet sun levels, in the radio enhanced regions, but local maxima were seen in the ECHs at the approximate locations of the

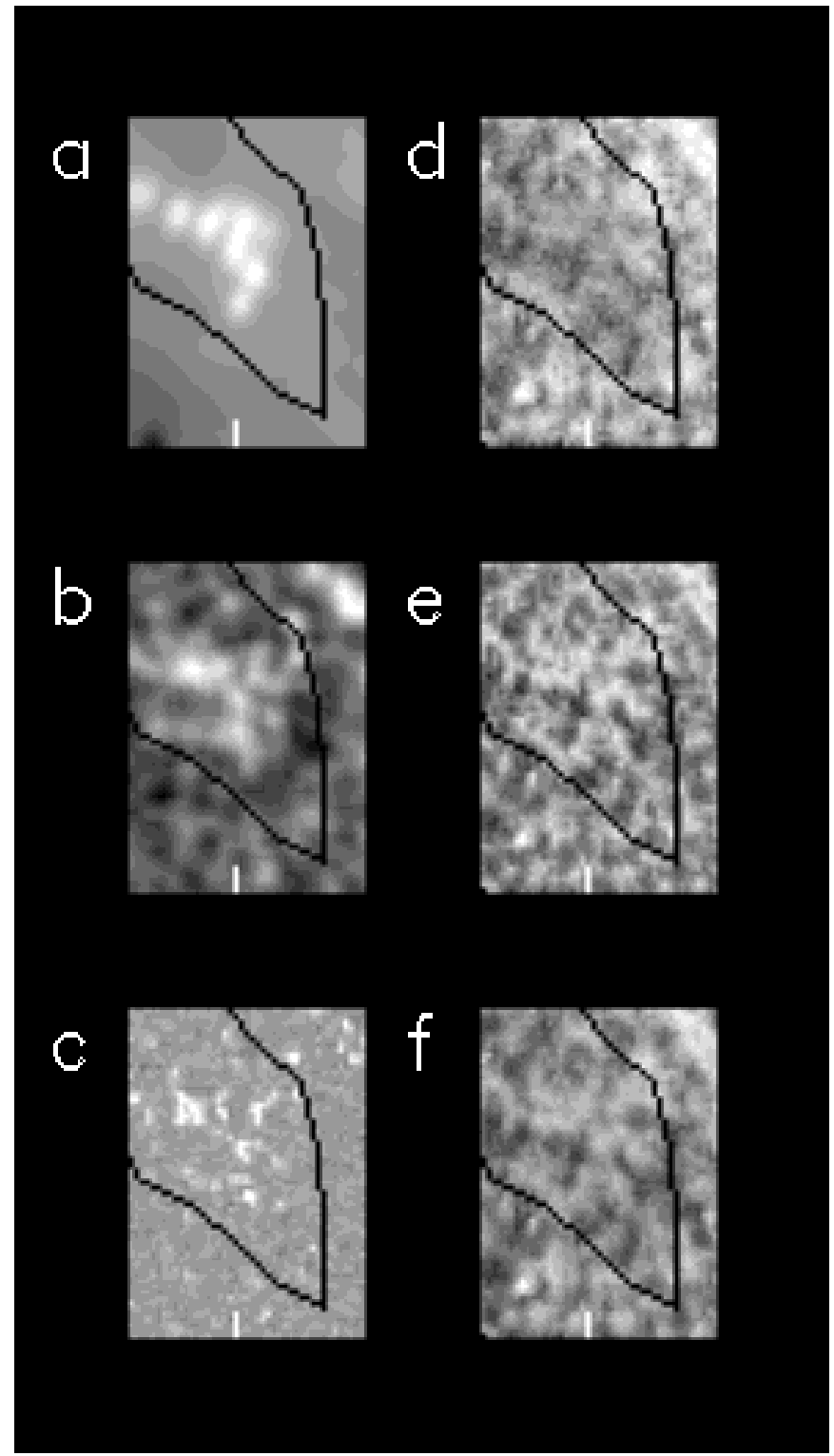

Fig. 2. SUMER field of view sub-frames of the October 24, 1999 observations. a) $17 \mathrm{GHz}$ radio data, b) re-binned, smoothed $\mathrm{H} \alpha$ image, c) magnetogram, and SUMER d) O I $948.7 \AA$, e) H i Ly $4949.7 \AA$, and f) He II 958.6 $\AA$ images.

enhancements. $\mathrm{H} \alpha$ images showed increased brightness in the ECH correlated with compact radio enhancements and strong unipolar magnetic fields. EUV Fe XII observations showed the presence of equatorial coronal plumes attached to some of the compact radio bright features.

\section{Spectral analysis of UV line emission}

From Table 1, it is evident that the SUMER ECH observations cover most of the electron temperature range from the upper chromosphere through the transition region with minimal gaps. If the ECH radio enhancements are due to a difference in the electron temperature height profile in the $10000 \mathrm{~K}$ to $630000 \mathrm{~K}$ range between the QS and portions of the ECH we would expect to see enhancements 
Table 1. Dates of the observations, emission lines, their wavelengths and formation temperatures, $T_{\mathrm{f}}$. Also listed are the types of regions in the fields of view the difference in ground and excited state energies, $\chi$, and a factor, $\alpha$, which is the ratio of the fractional change in brightness to the corresponding change in electron temperature. The $\alpha$ values for the optically thick Lyman lines were not computed and are not listed in the table.

\begin{tabular}{|c|c|c|c|c|c|c|}
\hline Date & Line & Wavelength/Å & $T_{\mathrm{f}} / \mathrm{K}$ & Area & $\chi / \mathrm{eV}$ & $\alpha$ \\
\hline $10 / 19 a$ & N II & 1084.5 & 27000 & $\mathrm{CH}+\mathrm{QS}$ & 11.4 & 4.5 \\
\hline$"$ & He II & 1085.5 & 47000 & $"$ & 52.2 & 12.4 \\
\hline$"$ & S III & 1077.1 & 63000 & $"$ & 12.9 & 1.9 \\
\hline$"$ & S IV & 1072.9 & 138000 & $"$ & 11.7 & 0.5 \\
\hline $10 / 19 b$ & $\mathrm{H}_{\text {I }} \mathrm{Ly} \beta$ & 1025.7 & 10000 & $\mathrm{CH}$ & 12.1 & - \\
\hline$"$ & C II & 1036.3 & 23000 & $"$ & 12.0 & 5.5 \\
\hline$"$ & O vi & 1031.9 & 288000 & $"$ & 12.0 & -0.02 \\
\hline $10 / 22$ & H п Ly $\beta$ & 1025.7 & 10000 & $\mathrm{CH}$ & 12.1 & - \\
\hline$"$ & C II & 1036.3 & 23000 & $"$ & 12.0 & 5.5 \\
\hline$"$ & O vi & 1031.9 & 288000 & $"$ & 12.0 & -0.02 \\
\hline $10 / 24$ & H I Ly 4 & 949.7 & 10000 & $\mathrm{CH}+\mathrm{QS}$ & 13.1 & - \\
\hline$"$ & O I & 948.7 & 10000 & $"$ & 13.1 & 14.0 \\
\hline$"$ & He II & 958.6 & 47000 & $"$ & 53.7 & 12.8 \\
\hline $10 / 29$ & Si II & 1533.4 & 14000 & $\mathrm{CH}+\mathrm{QS}$ & 8.1 & 6.2 \\
\hline$"$ & N IV & 765.1 & 140000 & $"$ & 16.2 & 0.84 \\
\hline$"$ & $\mathrm{Ov}$ & 760.4 & 230000 & $"$ & 26.5 & 0.84 \\
\hline$"$ & Ne VIII & 770.4 & 630000 & $"$ & 16.1 & -0.2 \\
\hline
\end{tabular}

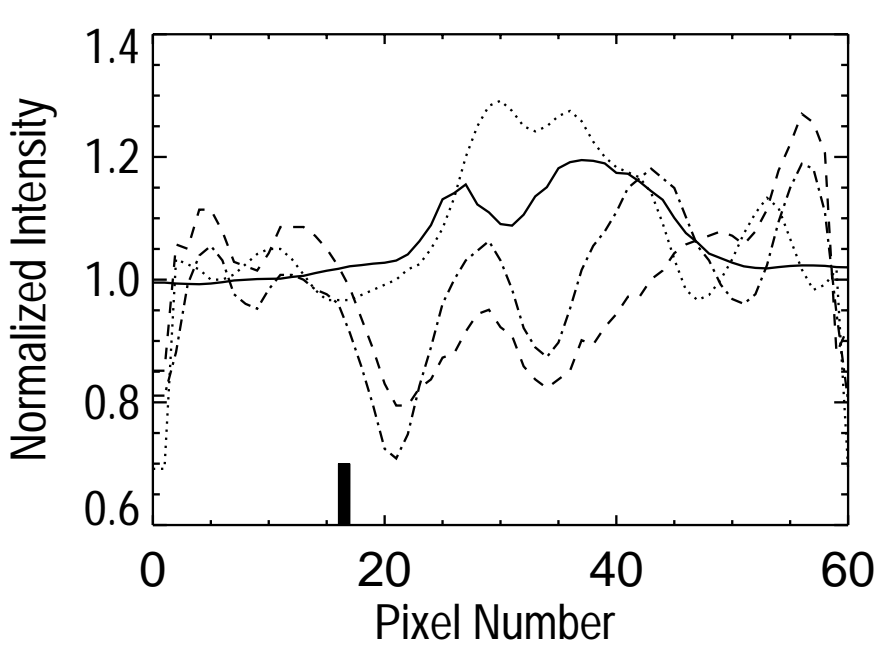

Fig. 3. Plots of the October 24 radio observations (solid), $\mathrm{H} \alpha$ (dotted), Hi Ly 4 (dashed) and HeII (dot-dash) brightness along pixel Col. 20, which is marked at the bottom by a vertical white dash in Figs. 2a-f. The ECH boundary is marked by a vertical dash on the abscissa.

in some of the UV lines measured. In fact, there are no corresponding differences in UV line intensity in any of the ECH radio enhancement sub-regions observed from October 19-28, 1999 beyond the normal network UV brightening (Wilhelm et al. 2001), however, as mentioned above relative maxima were observed. Increases in line width were measured in all of the chromospheric and transition region lines observed in the ECH network, consistent with previous observations (Lemaire et al. 1999). These increases are probably due to line-of-sight spicular motions and not directly related to the electron temperature, which determines radio brightness. Outflow velocities of 5 to $10 \mathrm{~km} \mathrm{~s}^{-1}$ were detected for lines formed above $100000 \mathrm{~K}$. The investigation demonstrated that ECHs were very similar to PCHs in all measurable aspects.

We may estimate the effect of temperature increases on the UV spectral lines observed with SUMER. The computation depends upon the line optical depth. For lines from optically thin regions, the brightness $B$ is computed by integrating the local emissivity $E$ along the line of sight:

$$
B=\int E \mathrm{~d} l
$$

where the integral is performed over the path $L$, which spans the radiating plasma. The local emissivity is given by:

$E=n_{\mathrm{s}} n_{\mathrm{e}} Q$

where $n_{\mathrm{e}}$ is the electron density, $n_{\mathrm{s}}$ is the species density and $Q$ is the excitation coefficient, computed by integrating the electron collisional excitation cross-section over the Maxwellian electron energy distribution, and therefore a function of electron temperature. The species density is given by: $n_{\mathrm{s}}=A_{\mathrm{E}} f_{\text {ion }} n_{\mathrm{e}}$, where $A_{\mathrm{E}}$ is the elemental abundance fraction and $f_{\text {ion }}$ is the ionization state fraction, also a function of electron temperature.

If $n_{\mathrm{e}}, Q, f_{\text {ion }}$ are taken to be the mean quantities along the effective path length rather than local quantities, the brightness is given by:

$B=A_{\mathrm{E}} n_{\mathrm{e}}^{2} f_{\text {ion }}(T) Q(T) L$

where the temperature dependence of $f_{\text {ion }}$ and $Q$ is explicitly noted.

The ionization fraction may be calculated by simultaneously solving a set of rate equations for the population of 
each ionization state. These calculations are involved and detailed results are not readily available. However, for the purpose of this study, we assume that all ions are at temperatures which are near the peak of their abundance versus temperature curves, and therefore the ionization fractions are not sensitive to the small temperature changes being considered here. While this might not hold for all the lines observed, this assumption allows an upper limit to be determined for any possible temperature increase. In the fixed abundance case, the temperature dependence of the line brightness is determined only by the excitation coefficient, $Q$.

The temperature dependence of $Q$ determines the ratio between the fractional changes in line brightness and electron temperature under the assumption of all other quantities remaining constant. This factor, denoted by $\alpha$, satisfies the relation:

$\frac{\mathrm{d} B}{B}=\alpha \frac{\mathrm{d} T}{T}$.

From Eqs. (3) and (4),

$\alpha=\frac{T}{Q} \frac{\mathrm{d} Q}{\mathrm{~d} T}$.

For a Maxwellian electron energy distribution and allowed dipole transitions, $Q$ is given by (McWhirter 1965):

$Q=C T^{-1 / 2} \exp (-\chi / k T)$

where $C$ is a constant dependent on atomic physics, $k$ is Boltzmann's constant and $\chi$ is the difference between upper state and lower state energies in the collisional excitation. The ratio of the fractional change in brightness to the fractional change in electron temperature is given by:

$\alpha=\frac{\chi}{k T}-\frac{1}{2}$.

All of the lines observed except for the hydrogen Lyman lines are assumed to be from optically thin regions. The ratios, $\alpha$, for these lines were computed and are listed in Table 1.

The range of $\alpha$ is from -0.02 to 14.0 , with the lowest values of -0.02 and -0.2 for lines formed at the highest temperatures, $288000 \mathrm{~K}$ and $630000 \mathrm{~K}$. For lines formed at temperatures between $10000 \mathrm{~K}$ and $230000 \mathrm{~K}$, we find $0.5<\alpha<14.0$, and therefore those lines are sensitive to changes in temperature. If the radio enhancement was caused by an increase in electron temperature of at least 15 to $20 \%$ at the formation height of the lines measured, there would be corresponding increase of at least $7.5 \%$ in line brightness for the least sensitive line and a factor of 14 increase in brightness for the most sensitive line. Since any increase in line brightness with respect to quiet sun levels is less than $2 \%$, the evidence is not strong for the optically thin case of higher electron temperatures in ECH radio enhanced regions. It is possible that the assumptions of constancy in $n_{\mathrm{e}}, L$, etc. are invalid, but it is highly unlikely that these other factors would change in such a way as to keep all of the line brightness constant under a significant temperature change.

For the nearly optically thick case, such as SUMER lines H I Ly $\beta$ and Ly 4 , the brightness $B$ is approximated by the Planck distribution:

$B=C \frac{\nu^{3}}{\mathrm{e}^{h \nu / k T}-1}$

where $C$ is a constant, $\nu$ is the photon frequency and $h$ is Planck's constant. When the photon energy, $h \nu$, is large compared with the thermal energy, $k T$, the brightness is very sensitive to temperature changes. In the case of the Ly $\beta$ and Ly 4 lines, $h \nu \approx 5 k T$, and a $1.5 \%$ change in temperature would cause a $15 \%$ change in brightness. These are rough estimates, since the conditions in question are not completely optically thick. Since no brightness increase was measured in the enhancement region, there is no evidence for an increased temperature at the $\operatorname{Ly} \beta$ and Ly 4 formation height, where the temperature is $20000 \mathrm{~K}$.

\section{4. $\mathrm{H} \alpha$ equatorial coronal hole enhancement}

In spite of the lack of an ECH enhancement in any of the UV lines observed, the two detections of $\mathrm{H} \alpha$ increased emission in ECHs coincident with radio bright spots were unequivocal. We examined the $\mathrm{H} \alpha$ images in an attempt to gain insight into the cause of the radio enhancements. The morphology of the enhanced $\mathrm{H} \alpha$ emission is apparent in the full resolution $\mathrm{H} \alpha$ image, which shows that the $\mathrm{H} \alpha$ enhancement results from three phenomena: the presence of bright fibrils and bright points, and the absence of dark fibrils. At full resolution, the $\mathrm{H} \alpha$ enhancements peak at a contrast of 1.8 above the QS level, rather than the peak value of 1.3 measured in the images which were smoothed to match the radio resolution. The contrast of the original image is greater, since the bright $\mathrm{H} \alpha$ fibrils have widths of $2^{\prime \prime}$ to $3^{\prime \prime}$, much narrower than the smoothing function width. The minimum brightness level in the enhancement lies at the average level outside the enhancement, due to the lack of dark fibrils. Since the fibrils are not fully resolved at the BBSO resolution, the true peak contrast may be greater than 1.8 , which suggests that the true peak radio enhancement may also be greater than 1.8. The source of the bright $\mathrm{H} \alpha$ fibrils in enhanced regions must be an increase in either intrinsic fibril brightness or filling factor. An increase in filling factor of 1.3 could explain the observation, and would occur if fibrils were either thicker or more densely packed. This seems plausible, since the well defined radio enhancements and $\mathrm{H} \alpha$ bright spots occur in regions of strong magnetic fields, distributed among many flux tubes, which are likely the bases of the fibrils observed in $\mathrm{H} \alpha$.

In order to test whether the increase in radio and $\mathrm{H} \alpha$ emission in the enhancements might be due to a filling factor increase, we computed the histograms of the radio and $\mathrm{H} \alpha$ emission in the area within both the $\mathrm{CH}$ and the SUMER field of view. If the enhancements are caused by a 


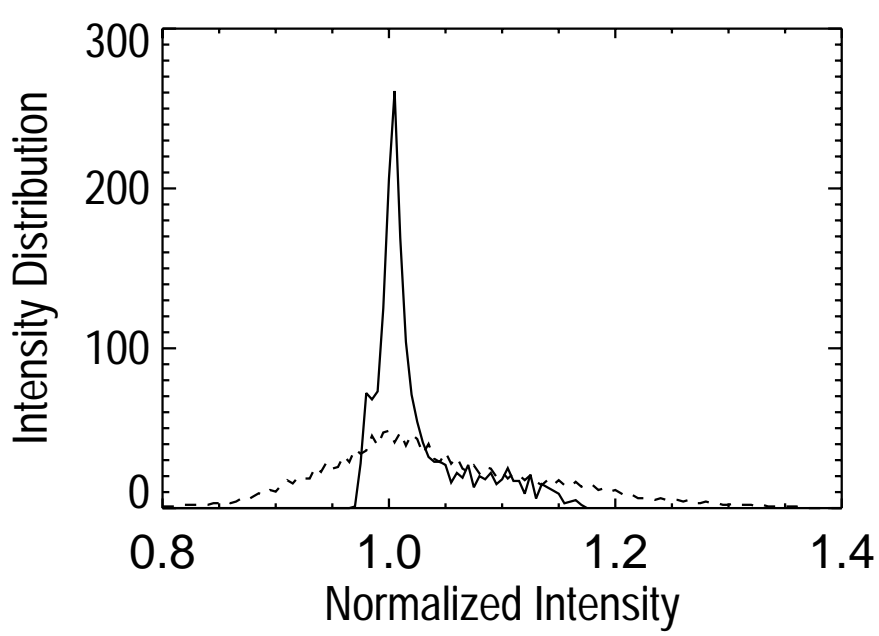

Fig. 4. Histograms of the $17 \mathrm{GHz}$ radio (solid) and $\mathrm{H} \alpha$ (dotted) emission in the area intersected by the $\mathrm{CH}$ boundary and SUMER field of view.

fibril filling factor increase, the integrated enhanced emission in both images should be equal. The background intensity in the images is normalized to unity, and both histograms are plotted in Fig. 4 . The $\mathrm{H} \alpha$ distribution extends to higher intensity, possibly due to insufficient smoothing of the $\mathrm{H} \alpha$ image. If the radio resolution element is larger than 19", a wider smoothing function should be used. Since the background and enhancement distributions overlap, we follow the method developed by Skumanich et al. (1975), to determine the proportion of integrated bright point emission. The distribution from zero to the intensity at the peak is reflected about the peak to create the background distribution, which is subtracted from the total to obtain the bright pixel distribution. Integrating the resulting distributions yields integrated enhanced emission fractions of $3.3 \%$ for $\mathrm{H} \alpha$ and $2.8 \%$ for $17 \mathrm{GHz}$ radio. Given the uncertainty in the technique used to obtain the bright pixel distributions, the two emission fractions might be equal. This result is consistent with a fibril filling factor increase causing both the radio and $\mathrm{H} \alpha$ enhancements.

To date, there are no $17 \mathrm{GHz}$ radio images of ECHs at resolution less than $\approx 18^{\prime \prime}$. However, higher resolution images of other types of solar regions have been obtained at several other frequencies. Images of a macrospicule on the limb at 4.8, 8.5 and $15 \mathrm{GHz}$ have been made using the Very Large Array (VLA) at 4" resolution (Habbal \& Gonzalez 1991), but no spicules are visible, since they would be unresolved. Microwave images of the QS on the disk have been obtained at $4.9 \mathrm{GHz}$ with $6^{\prime \prime}$ resolution (Gary \& Zirin 1988), 8.5 GHz at $4^{\prime \prime}$ resolution (Gary et al. 1990) and 19.9 and $22.5 \mathrm{GHz}$ at $3^{\prime \prime}$ and $5^{\prime \prime}$ resolution, respectively (Bastian et al. 1996). In all of these observations, radio fine structure at the resolution limit was present. The 4.9 and $8.4 \mathrm{GHz}$ images showed strong correlation with $\mathrm{H} \alpha$ images. Bright $\mathrm{H} \alpha$ fibrils and points corresponded to enhanced radio emission and magnetic flux. However, none of the microwave observations had sufficient resolution to resolve fibrils. The close correlation detected between 4.8 , and $8.5 \mathrm{GHz}$ emission and $\mathrm{H} \alpha$ emission is consistent with ECH $17 \mathrm{GHz}$ radio enhancements being caused by bright fibrils.

\section{Discussion and conclusion}

A series of SOHO/SUMER UV line spectral measurements were made during the passage of an ECH across the disk to search for evidence of higher chromospheric or transition region temperatures in areas of $\mathrm{CH}$ radio enhancements. Spectral analysis shows there is no significant electron temperature increase in the enhancement region relative to the quiet sun for temperatures between approximately $10000 \mathrm{~K}$ and $230000 \mathrm{~K}$. However, significant changes were found within the ECH between radio-enhanced and non-radio-enhanced regions. UV line emission was greater in the radio enhanced regions as compared with the ECH outside the enhancements. We need more observations in lines formed at $10000 \mathrm{~K}$ to arrive at firm conclusions regarding the physical conditions in radio enhancements at the levels of UV line formation.

Paradoxically, the only correlation of increased ECH emission (relative to the quiet sun) with radio enhancements was found with $\mathrm{H} \alpha$ and Fe XII emission. The compact radio enhancements coincided with the footpoints of coronal plumes as observed in Fe XII emission, but not with the plume structure above the base. H $\alpha$ emission is formed between $7000 \mathrm{~K}$ and $11000 \mathrm{~K}$ (Avrett 2000), while the Fe XII emission is formed at $1400000 \mathrm{~K}$. No increase above quiet sun levels was detected in lines formed between these temperature extremes. The radio enhancements cannot be caused directly by the mechanism which causes the Fe XII increase, since the radio brightness temperature is $10000 \mathrm{~K}$, and modeling of the coronal $17 \mathrm{GHz}$ radio contribution shows it is negligible (Gopalswamy et al. 1998, $1999 \mathrm{a}, \mathrm{b})$. The coronal plasma at $1000000 \mathrm{~K}$ above the radio enhancement formation layer must be heated by some form of energy flowing along magnetic flux tubes, such as wave flux, which would explain the correlation with radio enhancements.

Previous ECH microwave observations have led to speculations that the radio enhancements are caused by increased cross-field heat conduction or reconnection in the upper chromosphere, which would cause a temperature increase at the radio formation layer (Gopalswamy et al. 1998). The SUMER observations presented here provide no evidence of such a temperature increase relative to the quiet sun in the form of increased UV line emission. A higher transition region filling factor as cause of the ECH enhancement was also suggested (Gopalswamy et al. 1999a or b), but this would also cause increased UV line emission, which is not observed. Other spectral studies in the UV and FUV have shown higher inter-network chromospheric line emission, 6 to $7 \%$ greater than in nonhole regions, at several locations in ECHs for CaII K, Mg II K and H i Ly $\alpha$ (Bocchialini \& Vial 1996), but radio images were not available for comparison. Analysis of 
network chromospheric line profiles showed a smaller enhancement, 1 to $2 \%$. The area covered by the FUV observations reported here was relatively small, and therefore this increase would not be detectable. Since the radio and $\mathrm{H} \alpha$ enhancements occur within the network where fibrils are present, our results are consistent with these observations. However, ECH UV line observations with larger coverage of the disk would allow more accurate statistical comparisons between the enhancement and non-hole regions.

No well defined radio enhancements were found without corresponding $\mathrm{H} \alpha$ enhancements. This suggests that the cause of the radio enhancement may be in the middle to upper chromosphere, which is part of the $\mathrm{H} \alpha$ formation region. This is consistent with comparisons of polar radio enhancements and He II $304 \AA$ emission, formed at $80000 \mathrm{~K}$, which show a lack of correlation between the $17 \mathrm{GHz}$ radio and EUV bright regions (Nindos et al. 1999). The authors thus concluded that $17 \mathrm{GHz}$ microwave enhancements are formed below $80000 \mathrm{~K}$. In addition, studies of $17 \mathrm{GHz}$ polar emission scale heights show that radio brightenings lie below the $304 \AA$ limb, supporting a formation temperature below $80000 \mathrm{~K}$ (Nindos et al. 1999). These results agree with studies of $87 \mathrm{GHz} \mathrm{CH}$ enhancements which show no correlation with $304 \AA$ emission (Pohjolainen 2000). Since the $\mathrm{H} \alpha$ enhancement is due to increased fibril and bright point emission, the microwave enhancement is likely formed by bright radio fibrils and points. The ECH radio enhancement fibrils are bright either because they have a larger filling factor relative to non-hole fibrils or because the radio emission is formed at a higher temperature. In the $\mathrm{ECH}$ radio enhancements, the integrated bright emission in radio and $\mathrm{H} \alpha$ was approximately equal, suggesting a fibril filling factor increase in both cases. However, both phenomena might also be caused by a temperature increase, for example from $10000 \mathrm{~K}$ to $12000 \mathrm{~K}$ or higher at the radio formation height. Since the formation of the $\mathrm{H} \alpha$ line is complicated, we cannot predict with certainty how the line brightness would be affected. The lack of enhancement in upper chromospheric lines seems to weigh against a filling factor increase. Larger fibrils should cause more upper chromospheric line emission, unless there is a regulating mechanism which fixes the temperature and line-averaged density in that region, regardless of the energy passing through it.

High-resolution images of radio-enhanced $\mathrm{CH}$ regions, which could be made using the VLA, might reveal the structure of the ECH radio enhancements at $17 \mathrm{GHz}$. If the cause is bright fibrils, observations at $2^{\prime \prime}$ resolution or better would resolve them. The enhancements may be caused by the combination of concentrated flux tubes and reduced ECH pressure. Fibrils, which form along flux tubes, may have a slightly different structure in highfield ECH regions. Since the coronal pressure is lower, the pressure scale length between the center of the fibril and the corona may be longer, causing both the $17 \mathrm{GHz}$ radio and $\mathrm{H} \alpha$ emission to form at a higher temperature.
The density at $7000 \mathrm{~K}$ to $12000 \mathrm{~K}$ might be slightly higher in the enhancements, causing the radio and $\mathrm{H} \alpha$ emission to form at higher temperatures, since the optical depth is proportional to the square of the density. The densities in regions with temperature above $12000 \mathrm{~K}$ might be similar to non-hole regions, explaining the lack of enhancement in upper chromospheric and transition region lines. Modeling of fibrils in ECHs may help to explain the radio and $\mathrm{H} \alpha$ enhancements and lack of ECH UV line emission increases. Another explanation for the enhancements may be nonthermal phenomena. Brightness temperature fluctuations of $300 \%$ observed in another ECH were suggested to have a non-thermal cause (Gopalswamy et al. 1999a). However, the factor of three increase in microwave brightness could also be caused by a temporary factor of 2.2 increase in density.

Acknowledgements. The SUMER project is financially supported by the Deutsches Zentrum für Luft- und Raumfahrt (DLR), the Centre National d'Études Spatiales (CNES), the National Aeronautics and Space Administration (NASA), and the European Space Agency's (ESA) PRODEX programme (Swiss contribution). The work presented here was supported by AFOSR Grant No. USAF F49620-00-1-0012 and NASA Grant No. NAG5-9600 and carried out at the NASA Goddard Space Flight Center. The radio images were provided by the Nobeyama Radio Observatory, and the $\mathrm{H} \alpha$ images were provided by the Big Bear Solar Observatory, operated by the New Jersey Institute of Technology. The EIT and MDI data are by courtesy of the EIT and MDI consortia. We would like to thank Philippe Lemaire, who assisted in planning the SUMER observations, and Stuart Jordan of NASA/GSFC who provided useful comments on the manuscript. SOHO is an ESA/NASA collaborative mission.

\section{References}

Avrett, E. H. 2000, private communication

Bastian, T. S., Dulk, G. A., \& LeBlanc, Y. 1996, ApJ, 473, 539

DeForest, C., Hoeksema, J., Gurman, J., et al. 1997, Sol. Phys., 175,393

Bocchialini, K., \& Vial, J.-C. 1996, Sol. Phys., 168, 37

Delaboudinière, J.-P., Artzner, J., Brunaud, A., et al. 1995, Sol. Phys., 162, 291

Domingo, V., Fleck, B., \& Poland, A. I. 1995, Sol. Phys., 162, 1

Evanov, V., Labrum, N., Moiseev, I., Nesterov, N., \& Steward, R. 1980, Izv. Krymskoi Astrofiz. Obs., 61, 52

Fisher, R., \& Guhathakurta, M. 1995, ApJ, 447, L139

Furst, E., \& Hirth, W. 1975, Sol. Phys., 42, 157

Gary, D. E., et al. 1997, Bull. Amer. Astron. Soc., 29, 8.01

Gary, D. E., \& Zirin, H. 1988, ApJ, 329, 991

Gary, D. E., Zirin, H., \& Wang, H. 1990, ApJ, 355, 321

Gopalswamy, N. 2000, Proc. IAU Symp., 199, Pune, India

Gopalswamy, N., Shibasaki, K., DeForest, C., et al. 1998, Synoptic Solar Physics, ed. K. Balasubramaniam, J. Harvey, \& D. Rabin, ASP Conf. Ser., 140, 363

Gopalswamy, N., Shibasaki, B., Thompson, B., et al. 1999a, Solar Wind Nine, AIP, ed. S. Habbal, R. Esser, J. Hollweg, \& P. Isenberg, 277 
Gopalswamy, N., Shibasaki, B., Thompson, B., et al. 1999b, J. Geophys. Res., 104, 9767

Gopalswamy, N., Shibasaki, K., \& Salem, M. 2000, A\&A, in press

Grebinskii, A. 1987, Sov. Astron. Lett., 13, 299

Habbal, S. R., \& Gonzalez, R. D. 1991, ApJ, 376, L25

Huber, M., et al. 1974, ApJ, 194, L115

Kosugi, T., Ishiguro, M., \& Shibasaki, K. 1986, PASJ, 38, 1

Kundu, M., \& McCullough, T. 1972, Sol. Phys., 24, 133

Krieger, A. S., Timothy, A. F., \& Roelof, E. C. 1973, Sol. Phys., 29, 505

Lemaire, P., Bocchialini, K., Aletti, V., et al. 1999, Space Sci. Rev., 87, 249

McWhirter, R. 1965, Plasma Diagnostic Techniques, ed. R. Huddlestone, \& S. Leonard (Academic Press, New York)

Nakajima, H., Nishio, M., Enome, S., et al. 1995, JApAS, 16, 437
Nindos, A., et al. 1999, ApJ, 527, 415

Pohjolainen, S., Portier-Fozzani, F., \& Ragaigne, D. 2000, A\&A, 143, 227

Pohjolainen, S. 2000, A\&A, 361, 349

Shibasaki, K. 1998, ASP Conf. Ser., 140, 373

Skumanich, A., Smythe, C., \& Frazier, E. N. 1975, ApJ, 200, 747

Wefer, F., \& Bleiweiss, M. 1976, BAAS, 8, 338

Wilhelm, K., Curdt, W., Marsch, E., et al. 1995, Sol. Phys., 162,189

Wilhelm, K., Lemaire, P., Dammasch, I. E., et al. 1998, A\&A, 334,685

Wilhelm, K., Dammasch, I., \& Xia, L. 2001, Adv. Space Res., in press 